\title{
Validity Conditions in Agreement Problems and Time Complexity
}

$\mathbf{N}^{\circ} 4526$

August 2002

THÈME 1 



\title{
Validity Conditions in Agreement Problems and Time Complexity
}

\author{
Bernadette Charron-Bost* $\quad$ Fabrice Le Fessant ${ }^{\dagger}$ \\ Thème 1 - Réseaux et systèmes \\ Projets SOR \\ Rapport de recherche $\mathrm{n}^{\circ} 4526$ - August 2002 - 15 pages
}

\begin{abstract}
We study the time complexity of different agreement problems in the synchronous model with crash failures, by varying their validity condition. We first introduce a continuous class of agreement problems, the $\mathrm{k}-\mathrm{TAg}$ problems, which includes both the Uniform Consensus and Non-Blocking Atomic Commitment. We then exhibit a general early-deciding algorithm, that we instanciate to solve every problem of this class. The algorithm always achieves the previously established lower-bounds for early-deciding, showing that these lower-bounds are tight.
\end{abstract}

Key-words: distributed consensus, synchronous model, non-blocking atomic commitment, eraly-deciding algorithm, k-TAg, lower-bounds, validity conditions, time complexity

\footnotetext{
* LIX, École Polytechnique, 91128 Palaiseau Cedex, France

$\dagger$ INRIA Rocquencourt, B.P. 105, 78153 Le Chesnay Cedex, France
} 


\section{Conditions de validité pour les problèmes d'agrément et complexité en temps}

\section{Résumé :}

Nous étudions la complexité en temps de differents problèmes d'agrément dans le modèle synchrone avec pannes par crash, en utilisant différentes conditions de validité.

Nous présentons d'abord une classe continue de problèmes d'agrément, les problèmes k-TAg, qui contient à la fois le Consensus Uniforme et le Non-Blocking Atomic Commitment.

Nous exhibons alors un algorithme général de décision précoce, que nous instancions pour résoudre chaque problème de cette classe.

L'algorithme atteint toujours les bornes minimales établies précédemment pour les algorithmes de décision précoce, montrant que ces bornes sont bien atteintes.

Mots-clés : consensus distribué, modèle synchrone, non-blocking atomic commitment, algorithme de décision précoce, $\mathrm{k}-\mathrm{TAg}$, bornes, limites, conditions de validité, complexité en temps 


\section{Introduction}

Reaching agreement in a distributed system is a fundamental problem which arises in numerous guises. In all the versions of agreement problems, each process start with an initial value from some fixed set $V$. At some point in the computation, correct processes must irrevocably decide on some values in $V$. The specification of an agreement problem includes two safety conditions which basically depend on the failure model that is considered [10]. In this paper, we suppose that processes can fail only by crashing; for this failure model, all the agreement problems share the safety condition, called agreement, which specifies that no two processes may decide differently. Another safety condition, called validity, describes the decision values that are permitted. Validity condition varies according to the particular version

of agreement problem. For instance, Fischer, Lynch and Paterson [5] consider a weak form of validity, called non-triviality, which only specifies that there exist at least two runs with two different decision values. For the crash failure model, validity condition of the Consensus problem, that we call integrity, imposes that any decision value for any process is the initial value of some process. Integrity is inappropriate as a validity condition for the transaction commitment problem where the initial votes of all processes influence the decision. Indeed, the validity condition of the nonblocking atomic commitment ( $N B-A C$, for short) is unanimity: decide 1 (commit) only if every process initial value is 1 (yes), and decide 0 (abort) if some process starts with 0 (no) or a failure occurs.

In this paper, we investigate the relationships between different agreement problems. Indeed, slight modifications in the validity condition may yield considerable discrepancy between agreement problems. For instance, in synchronous systems Dwork and Moses [4] show that two rounds are always sufficient for solving the agreement problem of [5] with the integrity condition, while any Consensus algorithm tolerating $t$ failures requires $t+1$ rounds. In asynchronous systems Charron-Bost and Toueg [2] show that Consensus and NB-AC are incomparable in almost all environments: NB-AC is never reducible to Consensus, and Consensus is not reducible to NB-AC, except when only one process may fail. However, we observe that increasing the synchrony degree allows us to compare these two problems, and makes them closer and closer: In each of the partially synchronous models described in [3], NB-AC is harder than Consensus; indeed if a majority of processes is correct, then Consensus is solvable but not NB-AC. Moreover, in synchronous systems Consensus and NB-AC are both solvable no matter how many processes fail, and so are equivalent in terms of solvability. Actually they are equivalent in a stronger sense: in the presence of up to $t$ failures, $t+1$ rounds are sufficient and necessary in the worst case

$\mathrm{RR} \mathrm{n}^{\circ} 4526$ 
execution to solve as well Consensus as NB-AC [10]. One can refine this comparison by discriminating runs according to the number of failures $f$ that actually occur. Charron-Bost and Schiper [1] and subsequently Keidar and Rajsbaum [6] proved that both Consensus and NB-AC require at least $f+2$ rounds if $f$ is less than $t-1$, and only $f+1$ rounds if $f=t-1$ or $f=t$ (where $t$ is the maximum number of failures).

One may wonder whether these lower bounds for early deciding in Consensus and NB-AC algorithms are tight. An algorithm for Consensus is presented in [1] that achieves these lower bounds. Unfortunately, this algorithm cannot be easily adapted for the NB-AC problem. As noticed by Lamport in [9], "a [popular] belief is that a three-phase commit protocol à la Skeen [11] is needed" for solving NB-AC. It is not exactly clear what that means, but it seems to imply that at least three rounds are required in failure free runs. The $f+2$ lower bound would be thereby too weak in the case of NB-AC.

In this paper, we show that this intuition is incorrect: for that, we devise an algorithm for NB-AC which achieves the general lower bounds for early deciding of $[1,6]$. Even when refining the comparison in this way, Consensus and NB-AC remains two equivalent problems.

Another contribution of the paper is to introduce a natural extension of the NBAC problem, which we call the $k$-Threshold Agreement problem. It turns out that this problem is also a generalization of Consensus. In this way, we connect Consensus and NB-AC from the strict view point of problem specification. Actually, our algorithm for NB-AC is general enough to work for any $k$-Threshold Agreement problem. These agreement problems for various values of $k$ - in particular, Consensus and NB-AC - can be proved incomparable in asynchronous systems, but become equivalent in synchronous systems in the strong sense of early deciding.

\section{The Model}

In this paper, we consider the synchronous model for distributed systems described in [10] which we review briefly.

Let $\Pi$ be a fully connected graph with $n$ nodes. We consider some fixed message alphabet $M$, and we let null be a placeholder indicating the absence of message.

A synchronous algorithm $A$ is a collection of $n$ processes, one for each node of $\Pi$. Each process $p_{i}, i \in \Pi$, is defined by four components: a set of states states $i$, a subset start $_{i} \subseteq$ states $_{i}$ of initial states, a message-generation function msgs $_{i}$ map- 
ping states $s_{i} \times \Pi$ to a unique (possibly null message, and a state transition function trans $_{i}$ mapping states $_{i}$ and vectors indexed by $\Pi$ of messages to states $_{i}$.

An execution of the algorithm $A$ begins with each process in an arbitrary start state. Then each process $p_{i}$, in lock-step, repeatedly performs the following three steps: (1) apply $\mathrm{msgs}_{i}$ to the current state to determine the messages to be sent and send these messages, $(2)$ receive all the messages which are sent to $p_{i}$ during the previous step, and (3) apply trans $_{i}$ to the current state and the messages that are just received to obtain the new state. The combination of these three steps is called a round of $A$.

A run of $A$ models an execution of the entire system: it is defined by the collection of the initial states of processes, and the infinite sequence of rounds of $A$ that are executed.

We assume that links are reliable but processes can fail by crashing, that is by stopping somewhere in the middle of its execution. A process may crash before or during some instance of the steps described above. In particular, a process may succeed in sending only a subset of the messages specified to be sent. After crashing at a round, a process does not send any message during subsequent rounds. A process is said to be correct in a run if it does not crash; otherwise it is said to be faulty.

\subsection{Agreement Problems}

We now focus on the two well-known agreement problems that are Consensus and Non-Blocking Atomic Commitment.

The Consensus problem considers a fixed collection of processes which have each an initial value drawn from some domain $V$. Processes must eventually decide on the same value; moreover, the decision value must be the initial value of some process. The Non-Blocking Atomic Commitment (NB-AC) problem arises in distributed database systems to ensure the consistent termination of transactions. Each process that participate in the processing of a database transaction arrives at an initial opinion about whether to commit the transaction or abort it, and votes yes or no accordingly. Processes must eventually reach a common decision (commit or abort). The decision to commit may be reached only if all processes initially vote yes. In this case, commit must be decided if there is no failure.

When identifying yes and commit with 1 , and no and abort with 0 , it appears that binary Consensus and NB-AC are two very similar problems. They share the following two conditions:

Agreement: No two processes (whether correct or faulty) decide differently.

$\mathrm{RR} \mathrm{n}^{\circ} 4526$ 
Termination: All correct processes eventually decide.

Binary Consensus and NB-AC only differ in their validity conditions, i.e., the conditions describing the decision values that are permitted: in the Consensus problem, the possible decision values are related only to the initial values, contrary to the NB-AC problem for which the decision values depend on both input values and failure pattern. More precisely, the validity condition of Consensus is as follows:

Integrity: Any decision value for any process is the initial value of some process.

The validity condition of $\mathrm{NB}-\mathrm{AC}$ is:

\section{Unanimity:}

1. If any process starts with 0 , then 0 is the only possible decision value.

2. If all processes start with 1 and there is no failure, then 1 is the only possible decision value.

At this point, it is relevant to consider the weak validity condition introduced by Lamport in [7]:

Weak Validity: If there is no failure, then any decision value is the initial value of some process.

Obviously, the weak validity condition is weaker than the validity conditions of both Consensus and atomic commitment, but it is stronger than the one in [5] which only precludes the trivial protocols that always decide the same value. This latter condition, that we call non-triviality, is as follows:

Non-triviality: For every value $v \in\{0,1\}$, there is an execution in which some process decides $v$.

Binary Consensus and NB-AC specifications are clearly not comparable because of their incomparable validity conditions ${ }^{1}$. However, it is possible to link up these two problems from a syntactic standpoint. For that, we introduce the $k$-Threshold Agreement problem (the $k-T A g$ problem, for short) that is parametrized by some integer $k \in\{1, \cdots, n\}$. The termination and agreement conditions of $k$-TAg are the same as those of Consensus and NB-AC, and its validity condition is as follows:

\footnotetext{
${ }^{1}$ Furthermore, Charron-Bost and Toueg [2] showed that these two problems are also incomparable from an algorithmic point of view: NB-AC is not reducible to Binary Consensus, and vice-versa (except when the maximum number of failures is 1 ).
} 


\section{k-Validity:}

1. If at least $k$ processes start with 0 , then 0 is the only possible decision value.

2. If all processes start with 1 and at most $k-1$ failures occur, then 1 is the only possible decision value.

This new problem is a natural generalization of the atomic commitment problem 1-TAg is exactly NB-AC -, but turns out to be also a generalization of Consensus $n$-TAg actually corresponds to binary Consensus -. We have thereby defined a chain of problems which interpolates between NB-AC and binary Consensus. The original motivation for this generalization is purely theoretical: it is interesting to connect two incomparable problems by exploiting differences in validity conditions. But it is easy to imagine actual situations in which such a generalization could be natural: for example, it might be desirable for processes to enforce them to decide abort only if a majority of processes initially vote no, but to require that they commit if all of them initially vote yes as soon as a minority of processes are faulty.

\section{Lower Bounds for Time Complexity}

A fundamental result in synchronous systems [4] is that if $n \geq t+2$, then any Consensus algorithm that tolerates $t$ crashes must run $t+1$ rounds in some execution before processes have all decided. As noticed by Lynch [10], the proof of this result still works if one weakens the validity condition to the weak validity condition. So the $t+1$ lower bound also holds for NB-AC and more generally, for the all the $k$-TAg problems. However, it no more holds if one weakens validity to the non-triviality condition. Indeed, Dwork and Moses [4] device a simple algorithm achieving nontriviality, agreement, and termination in only two rounds.

These remarks, which concern the generalization of the worst case time complexity of Consensus to other agreement problems, also apply when one refines the analysis of time complexity by discriminating runs according to the number of failures that actually occurs. More precisely, Charron-Bost and Schiper [1] show the following lower bounds:

Theorem 3.1 Let $A$ be a Consensus algorithm which tolerates $t$ process crashes. For each $f, 0 \leq f \leq t$, there exists a run of $A$ with at most $f$ crashes in which at least one process decides not earlier than during round $f+2$ if $f \leq t-2$, and not earlier than during round $f+1$ otherwise.

$\mathrm{RR} \mathrm{n}^{\circ} 4526$ 
Afterwards, Keidar and Rajsbaum [6] showed the same lower bounds by an alternative method. The two proofs use different techniques but both clearly extend to the weak validity condition. As a consequence, this shows that each $k$-TAg problem, and in particular NB-AC ${ }^{2}$, requires at least $f+2$ rounds to decide if $0 \leq f \leq t-2$ and $f+1$ rounds if $t-1 \leq f \leq t$.

\section{A General Early Deciding Algorithm}

We now describe a general algorithm for the $k$-TAg problem that tolerates $t$ crashes and achieves the lower bounds of Theorem 3.1 for early deciding.

Our algorithm is based on a strategy borrowed from [8] for determining the time of decisions: each process $p_{i}$ maintains a variable Failed containing the set of processes that $p_{i}$ detects to have crashed. During a round, $p_{i}$ learns that $p_{j}$ has crashed if $p_{i}$ receives no message from $p_{j}$ at this round. At the end of every round, each process $p_{i}$ updates its variable Failed. If Failed remains unchanged during some round, namely if $p_{i}$ detects no new crash failure, then $p_{i}$ becomes ready to decide in this round. Any process that becomes ready at round $r$ is permitted to decide at round $r+1$ if $r \leq t-1$ or as early as round $r$ if $r=t$ or $r=t+1$; furthermore, it broadcasts a ready message at round $r+1$ both to inform other processes that it is ready to decide and to force the processes to become ready in turn.

To determine its decision value, each process maintains a vector $W$ of size $n$. Initially, every process sets its own component of $W$ to its initial value, and the other components to $\perp$. At each round $r \geq 1$, every process $p_{i}$ broadcasts its own component $W[i]$. Then it collects all the values it has just received into $W$; if $p_{i}$ does not receive a value from $p_{j}$, it simply sets $W[j]:=\perp$. Finally, $p_{i}$ sets its own component to a new value which depends on the round number $r$ and the values $p_{i}$ has received, that is $W[i]=\Phi(r, W)$ where $\Phi$ is an arbitrary function mapping $\{1, \cdots, t+1\} \times(V \cup\{\perp\})^{n}$ to $V$. A simple induction on the round number shows that no process ever applies $\Phi$ to $(\perp, \cdots, \perp)$ during any execution of the algorithm. The formal code of the algorithm is given in Figure 1.

In this section, we first prove that if all the processes invoke the same function $\Phi$ to adjust their own components in $W$, then agreement is guaranteed. This com-

\footnotetext{
${ }^{2}$ Interestingly, the validity condition of the NB-AC problem makes simpler the proof of the $f+2$ lower bound: following the proof scheme of [1], the existence of a bivalent initial configuration (with respect to the set of runs with initial failures) and the basis case $f=0$ directly result from the validity condition of NB-AC.
} 
mon function is then determined according to the validity condition to satisfy: in particular, we specify a function $\Phi$ that guarantees the $k$-validity property.

\subsection{Correctness Proof}

Theorem 4.1 For any mapping $\Phi:\{1, \cdots, t+1\} \times(V \cup\{\perp\})^{n} \rightarrow V$, the algorithm in Figure 1 satisfies the termination and agreement properties in a synchronous system with at most $t$ faulty processes. Moreover, the algorithm achieves the lower bounds of Theorem 3.1 for early deciding.

Proof: To prove this theorem, we use the following notation and terminology. The value of any variable $x$ at process $p_{i}$ just at the end of round $r$ is denoted by $x_{i}^{(r)}$. By extension, $x_{i}^{(0)}$ denotes the initial value of $x_{i}$. A round $r$ is quiescent with regard to process $p_{i}$, also noted $i$-quiescent, if $p_{i}$ detects no additional failure during round $r$.

For termination, consider a run of the algorithm in which $f$ processes actually crash $(0 \leq f \leq t)$. Every correct process $p_{i}$ detects $f$ crashes in this run, and so there is at least one round $r_{i}$ with $1 \leq r_{i} \leq f+1$ which is $i$-quiescent, i.e., Rec_Failed ${ }_{i}^{\left(r_{i}\right)}=$ Failed $_{i}^{\left(r_{i}\right)}$. Suppose $p_{i}$ has not yet decided at the end of round $r_{i}-1$; then we have halt $t_{i}^{\left(r_{i}-1\right)}=$ false. There are two cases to consider:

1. Process $p_{i}$ is not ready at the end of round $r_{i}-1$, that is ready $y_{i}^{\left(r_{i}-1\right)}=$ false. Since round $r_{i}$ is $i$-quiescent, $p_{i}$ necessarily becomes ready at round $r_{i}$, and so $p_{i}$ decides at the end of round $r_{i}+1$ if $r_{i} \leq t-1$ and at the end of round $r_{i}$ if $r_{i} \geq t$.

2. Process $p_{i}$ is ready at the end of round $r_{i}-1$. In this case, the algorithm enforces $p_{i}$ to decide the current value of $W_{i}[i]$ at the end of round $r_{i}$.

In any case, $p_{i}$ decides by the end of round $f+2$ if $0 \leq f \leq t-1$ and by the end of round $f+1$ if $f=t-1$ or $f=t$. This proves that the algorithm meets the lower bounds of Theorem 3.1, and subsequently the termination property.

For agreement, we first give a lemma which establishes a weak form of agreement among the processes that are ready. More precisely, the lemma states that two ready messages sent at the same round carry the same value.

Lemma 4.2 If two messages $(r e a d y, v)$ and $\left(r e a d y, v^{\prime}\right)$ are sent at the same round $r \geq 2$, then $v=v^{\prime}$.

$\mathrm{RR} \mathrm{n}^{\circ} 4526$ 
states $_{\mathbf{i}}$

rounds $\in N$, initially 0

$W \in(V \cup\{\perp\})^{n}$, initially $(\perp, \cdots, v, \cdots, \perp)$ where $v$ is $p_{i}$ 's initial value

ready, a Boolean, initially false

halt, a Boolean, initially false

Rec_Failed $\subseteq \Pi$, initially $\emptyset$

Failed $\subseteq \Pi$, initially $\emptyset$

decision $\in V \cup\{$ unknown $\}$, initially unknown

\section{$\mathbf{m s g s}_{\mathbf{i}}$}

if $\neg$ halt then

if $\neg$ ready then send $W[i]$ to all processes

else send (ready, $W[i]$ ) to all processes

\section{$\operatorname{trans}_{\mathbf{i}}$}

if $\neg$ halt then

rounds $:=$ rounds +1

let $w_{j}$ be the value sent by $p_{j}$, for each $p_{j}$ from which a message arrives

if a message has arrived from $p_{j}$ then $W[j]:=w_{j}$

else $W[j]:=\perp$

if ready then

if rounds $\leq t$ then decision $:=W[i]$

halt $:=$ true

else if some message (ready,w) arrives then

$W[i]:=w$

ready $:=$ true

else Rec_Failed $:=$ Failed

Failed $:=\left\{p_{j}:\right.$ no message arrives from $p_{j}$ at the current round $\}$

$W[i]:=\Phi($ rounds,$W)$

if Rec_Failed $=$ Failed then ready $:=$ true

if ready and rounds $\geq t$ then decision $:=W[i]$

Figure 1: A General Algorithm for Early Deciding 
Proof: The proof is by induction on $r$. Let $p_{i}$ and $p_{j}$ be the senders of $(r e a d y, v)$ and $\left(\right.$ ready, $\left.v^{\prime}\right)$, respectively; from the algorithm, $p_{i}$ and $p_{j}$ have not yet halted but have both become ready at the end of round $r-1$.

Basis: $r=2$. Processes $p_{i}$ and $p_{j}$ can send a ready message at round 2 only if the first round is $i$ and $j$-quiescent. In other words, both $p_{i}$ and $p_{j}$ receive the same vector $W$ of $n$ initial values at round 1 , and so $W_{i}[i]^{(1)}=W_{j}[j]^{(1)}=\Phi(1, W)$. From the code of the algorithm, $p_{i}$ and $p_{j}$ send ready messages at round 2 which are tagged by $W_{i}[i]^{(1)}$ and $W_{j}[j]^{(1)}$, respectively. Therefore, we have $v=v^{\prime}$.

Inductive step: Assume $r>2$, and suppose the lemma holds at any round $r^{\prime}$ with $2 \leq r^{\prime}<r$. There are three cases to consider:

1. Processes $p_{i}$ and $p_{j}$ both receive some ready messages at round $r-1$. From the inductive hypothesis, all these ready messages are tagged by the same value $\nu$. Upon receiving $(r e a d y, \nu)$ at round $r-1, p_{i}$ and $p_{j}$ set $W_{i}[i]:=\nu$ and $W_{j}[j]:=\nu$, respectively. At round $r, p_{i}$ and $p_{j}$ both broadcast ready messages which carry the current value of $W_{i}[i]$ and $W_{j}[j]$, i.e., the value $\nu$. Therefore, we have $v=\nu=v^{\prime}$.

2. Exactly one of the two processes, say $p_{i}$, receives some ready messages at round $r-1$; the same argument as before shows that each of them are tagged with value $\nu$. Let $p$ be the sender of such a message; process $p$ is obviously alive at the very beginning of round $r-2$. On the other hand, since $p_{j}$ becomes ready at round $r-1$ without receiving a ready message, it follows that round $r-1$ is $j$-quiescent. At round $r-1$, process $p_{j}$ thus detects no new failure and so receives a message from $p$. This message is equal to $($ ready, $\nu)$ since $p$ sends the same message to all processes. This contradicts that only $p_{i}$ receives a ready message at round $r-1$, and so this case cannot occur.

3. None of processes $p_{i}$ and $p_{j}$ receive ready messages at round $r-1$. From the algorithm, it follows that round $r-1$ is both $i$ and $j$-quiescent. Then $p_{i}$ and $p_{j}$ receive the same information at this round, and so the vectors $W_{i}$ and $W_{j}$ are equal just after the receive phase of round $r-1$. Let $W$ denote this vector. Since neither $p_{i}$ nor $p_{j}$ receive a ready message at round $r-1$, both $p_{i}$ and $p_{j}$ set their own component to $\Phi(r-1, W)$, that is $W_{i}[i]^{(r)}=W_{j}[j]^{(r)}=\Phi(r-1, W)$. Then $p_{i}$ and $p_{j}$ tag the ready messages that they send at round $r$ with the current values of $W_{i}[i]$ and $W_{j}[j]$, respectively. Therefore, we have $v=W_{i}[i]^{(r-1)}$, $v^{\prime}=W_{j}[j]^{(r-1)}$, and so $v=v^{\prime}$ as needed.

Lemma 4.2 
We are now in position to prove that the algorithm in Figure 1 satisfies agreement. Let $r$ be the first round in which some process decides. Let $p_{i}$ denote this process and $v$ its decision value. Let $p_{j}$ be a process that decides $v^{\prime}$ at round $r^{\prime}$. Thus, we have $r^{\prime} \geq r$. We consider two cases:

1. Process $p_{i}$ is ready at the end of round $r-1$, and so sends a ready message at round $r$. From the algorithm, this message is tagged by $W_{i}[i]^{(r-1)}$. Afterwards, $p_{i}$ does not modify the value of $W_{i}[i]$, and so decides $v=W_{i}[i]^{(r-1)}$. The $(r e a d y, v)$ message from $p_{i}$ is received by $p_{j}$ since $p_{j}$ is still alive at the end of round $r^{\prime}$, and $r^{\prime} \geq r$.

(a) If $p_{j}$ is also ready at the end of round $r-1$, then it sends a ready message at round $r$. Therefore, $r=r^{\prime}$ and the ready message from $p_{j}$ is tagged by the current value of $W_{j}[j]^{(r-1)}$. As for $p_{i}$, process $p_{j}$ decides value $v^{\prime}=W_{j}[j]^{(r-1)}$. Lemma 4.2 shows that $W_{i}[i]^{(r-1)}=W_{j}[j]^{(r-1)}$, and so $v=v^{\prime}$.

(b) Otherwise, $p_{j}$ receives some ready messages at round $r$, and by Lemma 4.2 all these messages are tagged by $v$. From the algorithm, $p_{j}$ must set $W_{j}[j]$ to $v$ and becomes ready at the end of round $r$. Then $p_{j}$ decides the current value of $W_{j}[j]$ at round $r$ or $r+1$. This implies that $v^{\prime}=v$, as needed.

2. Process $p_{i}$ is not ready at the end of round $r-1$. This may happen only if $r \geq t$, i.e., $r=t$ or $r=t+1$. Moreover, we can assume that $p_{j}$ is also not ready at the end of round $r-1$ (otherwise, we have $r^{\prime}=r$ and then we go back to the first case by exchanging the roles of $p_{i}$ and $p_{j}$ ). Therefore, none of the rounds $1, \cdots, r-1$ are $i$ or $j$-quiescent. There are two sub-cases to consider:

(a) Process $p_{i}$ receives some ready messages at round $r$. Then all these messages are tagged by the decision value $v$ of $p_{i}$. Let $p_{k}$ be the sender of such a message.

i. Assume process $p_{j}$ also receives $(r e a d y, v)$ from $p_{k}$ at round $r$; then $p_{j}$ becomes ready and sets $W_{j}[j]$ to $v$. Since $r \geq t, p_{j}$ decides at the end of round $r$ and its decision value is the current value of $W_{j}[j]$, i.e., $W_{j}[j]^{(r)}=v$. This proves that $v^{\prime}=v$.

ii. Otherwise process $p_{j}$ receives no message from $p_{k}$ at round $r$. Since $p_{k}$ sends a message to $p_{i}$ at this round, it necessarily succeeds in sending a message to $p_{j}$ at the previous round $r-1$. Therefore, round $r$ is not $j$-quiescent, and so $p_{j}$ does not decide at round $r$. Moreover, $p_{j}$ 
has detected at least $r$ crashes at the end of round $r$. Since $r \geq t$, no additional failure can occur later. This shows that process $p_{i}$ (that is still alive at the end of round $r$ ) is actually correct, and that it succeeds in sending $(r e a d y, v)$ to $p_{j}$ at round $r+1$. It follows that $p_{j}$ decides the value $v^{\prime}=v$ at round $r+1$.

(b) Process $p_{i}$ receives no ready message at round $r$. From the algorithm, it follows that round $r$ is $i$-quiescent.

i. If process $p_{j}$ receives a ready message at round $r$, then $p_{j}$ becomes ready and since $r \geq t$, it decides at round $r$. We get $r^{\prime}=r$, and by exchanging the roles of $p_{i}$ and $p_{j}$ we go back to the previous sub-case.

ii. Otherwise process $p_{j}$ receives no ready message at round $r$. At this point there are two possible cases:

A. Round $r$ is also $j$-quiescent. The algorithm enforces $p_{j}$ to decide at round $r$, and so $r^{\prime}=r$. The fact that round $r$ is both $i$ and $j$-quiescent ensures that $p_{i}$ and $p_{j}$ receive the same information at this round, and so the vectors $W_{i}$ and $W_{j}$ are equal just after the receive phase of round $r$. Let $W$ denote this vector. Since neither $p_{i}$ nor $p_{j}$ receive a ready message at round $r$, both $p_{i}$ and $p_{j}$ set their own component to $\Phi(r, W)$, that is $W_{i}[i]^{(r)}=$ $W_{j}[j]^{(r)}=\Phi(r, W)$. Then $p_{i}$ and $p_{j}$ decide on $v=W_{i}[i]^{(r)}$ and $v^{\prime}=W_{j}[j]^{(r)}$, respectively. This enforces that $v=v^{\prime}$.

B. Otherwise, $p_{j}$ detects a new crash at round $r$. Therefore, $p_{j}$ has detected at least $r$ crashes at the end of round $r$. We then argue as in a previous case. Since $r \geq t$, no additional failure can occur later. This shows that process $p_{i}$ (that is still alive at the end of round $r$ ) is actually correct, and that it succeeds in sending $($ ready, $v)$ to $p_{j}$ at round $r+1$. It follows that $p_{j}$ decides the value $v^{\prime}=v$ at round $r+1$.

$\square_{\text {Theorem } 4.1}$

We now address the validity issue. For that, we limit the set to which functions $\Phi$ belong. More precisely, for each $k \in\{1, \cdots, n\}$ we restrict ourselves to functions $\Phi$ which satisfy the following $\left(\mathcal{C}_{k}\right)$ condition: 
(1) $\Phi(r, W)=0 \quad$ if $r=1$ and $|\{i: W[i] \in\{0, \perp\}\}| \geq k$;

(2) $\Phi(r, W) \in\{W[i]: W[i] \neq \perp\} \quad$ otherwise and $W \neq(\perp, \cdots, \perp)$.

Note that for every $k \in\{1, \cdots, n\}$, there exist functions which satisfy $\left(\mathcal{C}_{k}\right)$. Moreover, part (2) in $\left(\mathcal{C}_{k}\right)$ is quite natural here since $\Phi$ is never applied to $(\perp, \cdots, \perp)$ during the algorithm (cf. Section 4).

We now prove that condition $\left(\mathcal{C}_{k}\right)$ for function $\Phi$ in Figure 1 yields an algorithm which solves the $k$-TAg problem.

Theorem 4.3 For any function $\Phi:\{1, \cdots, t+1\} \times(V \cup\{\perp\})^{n} \rightarrow V$ verifying $\left(\mathcal{C}_{k}\right)$, the algorithm in Figure 1 satisfies the $k$-validity property.

Proof: First suppose that at least $k$ processes start with the initial value 0 . Let $p_{i_{1}}, \cdots, p_{i_{k}}$ denote such processes, and let $p_{i}$ be any process. Process $p_{i}$ receives value 0 or no message at the first round from at least $k$ processes. From the definition of $\Phi_{k}(1,-)$, it follows that $W_{i}[i]^{(1)}=0$. The algorithm then enforces any component $W_{j}[j]$ to remain equal to 0 at the subsequent rounds. So, 0 is the only possible decision value.

On the other hand, consider a run of the algorithm in which all the processes start with 1 , and with at most $k-1$ failures. At the first round, every process $p_{i}$ receives at least $n-k+1$ messages with value 1 , and so $W_{i}[i]^{(1)}=1$ (if $p_{i}$ is still alive at the end of round 1). A simple induction on $r$ shows that for any $r$, we have $W_{i}[i]^{(r)}=1$ because of the definition of $\Phi_{k}(r,-)$ for $r \geq 2$. Therefore, the only possible decision value in this run is 1 .

Theorem 4.3

For $k=n$, the algorithm in Figure 1 thereby solves binary consensus. Obviously, this algorithm can be generalized for an arbitrary value set $V$.

Part 2 of $\left(\mathcal{C}_{k}\right)$ provides a large flexibility for the choice of $\Phi$. For instance, $\min , \max$, and $W[i]$ where $i=\min \{j: W[j] \neq \perp\}$ all yield correct solutions of the $k$ TAg problems. In the Non-Blocking Atomic Commitment case, this flexibility can be used to tune the frequency of commit decisions. Indeed, setting $\Phi(r, W)=\max _{i} W[i]$ in part 2 of $\left(\mathcal{C}_{k}\right)$ allows processes to commit more often: if all processes initially vote yes, then the decision is commit as soon as at least one correct process has received yes from all processes at the first round no matter whether failures occur. This is clearly a desirable feature of any database transaction system. 


\section{References}

[1] B. Charron-Bost and A. Schiper. Uniform consensus is harder than consensus. Technical Report DSC/2000/028, Département Systèmes de Communication, EPFL, May 2000.

[2] Bernadette Charron-Bost and Sam Toueg. Comparing the atomic commitment and consensus problems. In preparation, January 2001.

[3] C. Dwork, N. A. Lynch, and L. Stockmeyer. Consensus in the presence of partial synchrony. Journal of the ACM, 35(2):288-323, April 1988.

[4] Cynthia Dwork and Yoram Moses. Knowledge and common knowledge in a Byzantine environment: Crash failures. Information and Computation, 88(2):156-186, October 1990.

[5] M. J. Fischer, N. A. Lynch, and M. S. Paterson. Impossibility of distributed consensus with one faulty process. Journal of the ACM, 32(2):374-382, April 1985.

[6] I. Keidar and S. Rajsbaum. On the cost of fault-tolerant consensus when there are no faults - a tutorial. Technical Report MIT-LCS-TR-821, Laboratory for Computer Science, Massachusetts Institute of Technology, May 2001.

[7] Leslie Lamport. The weak byzantine generals problem. Journal of the ACM, 30(3):668-676, July 1983.

[8] Leslie Lamport and Michael Fischer. Byzantine generals and transaction commit protocols. Technical Report 62, SRI International, April 1982.

[9] Lamport Leslie. Lower bounds on consensus. March 2000.

[10] N. A. Lynch. Distributed Algorithms. Morgan Kaufmann, 1996.

[11] Dale Skeen. Nonblocking commit protocols. In Proceedings of the ACM SIGMOD Conf. on Management of Data, pages 133-147. ACM, June 1982.

$\mathrm{RR} \mathrm{n}^{\circ} 4526$ 


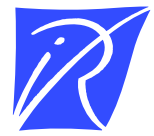

Unité de recherche INRIA Rocquencourt Domaine de Voluceau - Rocquencourt - BP 105 - 78153 Le Chesnay Cedex (France)

Unité de recherche INRIA Lorraine : LORIA, Technopôle de Nancy-Brabois - Campus scientifique 615, rue du Jardin Botanique - BP 101 - 54602 Villers-lès-Nancy Cedex (France)

Unité de recherche INRIA Rennes : IRISA, Campus universitaire de Beaulieu - 35042 Rennes Cedex (France)

Unité de recherche INRIA Rhône-Alpes : 655, avenue de l'Europe - 38330 Montbonnot-St-Martin (France)

Unité de recherche INRIA Sophia Antipolis : 2004, route des Lucioles - BP 93 - 06902 Sophia Antipolis Cedex (France)

INRIA - Domaine de Voluceau - Rocquencourt, BP 105 - 78153 Le Chesnay Cedex (France)

http://www.inria.fr

ISSN 0249-6399 